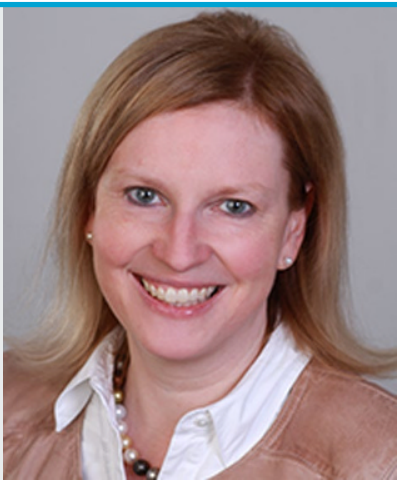

॥ Die Nachwuchsförderung der DGN liegt mir am Herzen

Prof. Dr. Christine Klein

Institut für Neurogenetik, Universitätsklinikum Schleswig-Holstein,

Universität Lübeck

Maria-Goeppert-Straße 1, 23562 Lübeck, Deutschland

christine.klein@neuro.uni-luebeck.de

\title{
DGNeurologie und neue Medien - ein interaktiver Dialog zu Chancen und Herausforderungen in unserem Fach
}

Liebe Kolleginnen und Kollegen,

in der nunmehr vierten Ausgabe unserer DGNeurologie möchte ich Sie als neue Präsidentin unserer Fachgesellschaft herzlich grüßen und mich an dieser Stelle vielmals für Ihr Vertrauen bedanken.

Mit dem Rückenwind der vielen positiven Entwicklungen in der Deutsche Gesellschaft für Neurologie (DGN) durch die Arbeit meines Vorgängers Prof. Gereon R. Fink - exemplarisch seien nur Erfolge wie die Gründung der DGN-Stiftung sowie unserer beiden neuen Zeitschriften genannt - freue ich mich sehr auf die vor mir liegende 2-jährige Amtszeit. Mit Prof. Peter Berlit konnten wir im vergangenen Jahr nicht nur einen hervorragenden Herausgeber für die DGNeurologie gewinnen, sondern auch einen tatkräftigen Generalsekretär für unsere Fachgesellschaft. Die hiermit nun mögliche größere Kontinuität in der Vorstandsarbeit ist überaus förderlich für die vielen wichtigen und häufig auch langfristig angelegten Aufgaben, wie z. B. die Verbesserung der Pflegesituation.

Mir liegen für meine Präsidentschaft 3 Themen besonders am Herzen: Zunächst möchte ich gern den Nachwuchs weiterhin für unser spannendes Fach begeistern und an die bereits hervorragenden Ergebnisse der Nachwuchsförderung der DGN anknüpfen. Wir haben hierbei besonderes Glück, da wir in unserer Gesellschaft so aktive „junge Neurologen“ (Sprecherin: Frau Dr. S. Aschenberg) haben. Im vergangenen Jahr entstanden aufgrund einer umfangreichen Weiterbildungsumfrage eine Berufsstarterbroschüre und ein sog. Weiterbildungs-Check. In diesem Jahr soll die inzwischen europaweit angelegte Umfrage zur neurologischen Weiterbildung abgeschlossen werden. Inspiriert zum einen durch diese Aktivitäten sowie weiterhin durch eine Einladung der „American Neurological Association“, bei der wir als Gäste an der Jahrestagung in Atlanta im Herbst 2018 unsere DGN re-

DGNeurologie 2019 • 2 (2): 77-79

https://doi.org/10.1007/s42451-019-0057-3

๑) Springer Medizin Verlag GmbH, ein Teil von Springer Nature 2019 präsentieren konnten, planen wir bei der DGN ein neues ,international outreach program": Jedes Jahr sollen ein europäisches Gastland ausgewählt, Vertreter der jeweiligen Fachgesellschaft zur DGN-Tagung eingeladen und ein internationaler Austausch auf der Ebene des neurologischen Nachwuchses etabliert werden.

Mein zweiter Interessenschwerpunkt für die Präsidentschaft liegt auf dem Gebiet der seltenen Erkrankungen. Von den mindestens 5000 bekannten seltenen Erkrankungen manifestieren sich geschätzt $80 \%$ in irgendeiner Form am Nervensystem. Die seltenen Erkrankungen können daher ohne Übertreibung als eine Domäne der Neurologie bezeichnet werden. Dies ist umso bedeutsamer, da es gerade in diesem Feld, besonders in den Bereichen Neurogenetik und Neuroimmunologie, bahnbrechende Fortschritte in Diagnostik und Therapie gibt.

Diese Fortschritte wurden nicht zuletzt durch enorme Anstrengungen und Erfolge in der neurowissenschaftlichen Forschung möglich gemacht. Deutschland als Standort für innovative und translationale Neuroforschung weiter zu stärken und möglichst viele „clinician scientists“ auszubilden - was keineswegs auf die akademischen Neurologien beschränkt sein darf ist mir ein weiteres wichtiges Anliegen.

Ich bin davon überzeugt, dass die vielen wichtigen Aufgaben nur gemeinsam gelöst werden können und dass es hierfür von größter Bedeutung ist, in einen engen, gemeinsamen Dialog einzutreten und in einem ersten Schritt zunächst eine repräsentative Datenbasis zu schaffen, mit deren Hilfe wir die wichtigsten Chancen und Herausforderungen in unserem Fach identifizieren können. Hierzu legte die DGN, basierend auf einer Idee von Prof. Pramstaller und in Umsetzung durch die mit dem Grimme-Preis ausgezeichneten Gebrüder Beetz in Berlin das Projekt „reimagine medicine" auf. Es handelt sich dabei um ein interaktives, crossmediales Veränderungsprojekt mit und durch uns Neurologen zu aktuellen Themen der Neurologie und auch der Medizin allgemein. Bitte nehmen Sie sich 10-15 min Zeit und laden Sie möglichst viele Kollegen ein, ebenfalls mitzumachen (https://re-imagine-medicine.de/\#screen/start). Bisher nahmen 1300 Mitglieder teil; unser Ziel ist es, möglichst viele Stimmen unserer Mitglieder einzubeziehen, was dem Projekt zugleich eine politische Wirkkraft verleiht. Wir sind sehr gespannt auf die Er- 
gebnisse, die wir in Stuttgart auf der DGN-Jahrestagung vorstellen werden und freuen uns auf den Dialog mit Ihnen allen und die gemeinsame Erarbeitung von Lösungen.

Schon im Voraus danke ich Ihnen herzlich fürs Mitmachen!

Ihre

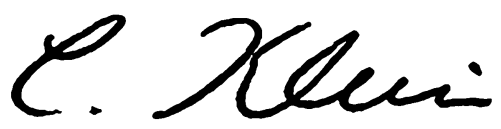

Christine Klein

Interessenkonflikt. C. Klein ist medizinische Beraterin bei Centogene und Biogen.

\section{Aktuelle Neuerscheinung}

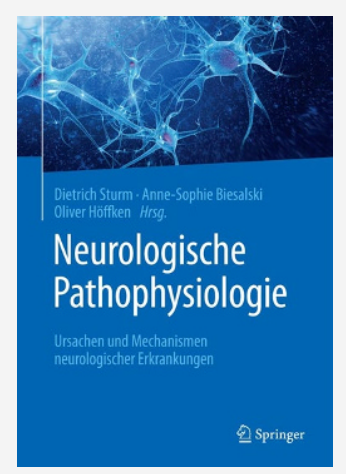

\section{Neurologische Pathophysiologie.}

Ursachen und Mechanismen neurologischer Erkrankungen

Dietrich Sturm, Anne-Sophie Biesalski, Oliver Höffken (Hrsg.)

1. Aufl. 2019, 274 S., 131 Abb.

Softcover 59,99€, ISBN 978-3-662-56783-8

In der klinischen Praxis der Neurologie ist es hoch relevant, neben dem neurologisch-topischen Wissen auch die pathophysiologischen Grundlagen neurologischer Erkrankungen zu beherrschen. Während das Medizinstudium die Kenntnis der Neuroanatomie als Basis für die klinisch-neurologische Diagnostik vermittelt, wird der Aspekt der Pathophysiologie häufig vernachlässigt.

Dieses Buch beleuchtet Mechanismen wichtiger neurologischer Erkrankungen, um pathophysiologische und biochemische Ursachen zu begreifen und sinnvoll in den klinischen Gesamtzusammenhang einordnen zu können.

Maßgeblich von „Jungen Neurologen" geschrieben, wendet es sich insbesondere an Ärztinnen und Ärzte in der neurologischen Weiterbildung sowie an interessierte Studierende. 
Hier steht eine Anzeige.

\section{Springer}

